Eur J Nucl Med (2002) 29:967-969

DOI 10.1007/s00259-002-0840-3

Published online: 17 May 2002

(C) Springer-Verlag 2002

\section{Which attenuation coefficient to use in combined attenuation and scatter corrections for quantitative brain SPET?}

\section{Dear Sir,}

We read with interest the recent paper by Shiga et al. published in the European Journal of Nuclear Medicine [1]. The authors report some interesting results concerning the effect of triple-energy window-based scatter correction on brain perfusion SPET using statistical parametric mapping (SPM) analysis. The research performed is worthwhile and contributes significantly to our understanding of the effect of scatter correction; to the best of our knowledge, it is also the first time that SPM analysis has been used for this purpose instead of conventional qualitative and region of interest (ROI)-based quantitative evaluations [2]. However, we feel that certain relevant issues were not sufficiently addressed by the authors, and we would like to make some comments on this work.

The variety of pertinent publications in this field emphasises the importance of methodological considerations. Unfortunately, there are a considerable number of references relating to the subject that were not cited in the paper (e.g. [3, 4, 5, 6, 7, 8, 9, 10, 11, 12, 13]). In our opinion, the reader would have gained a clearer picture of research performed in the field if these references had been cited and discussed. Firstly, the way in which the authors perform combined attenuation and scatter correction is not well elucidated in the Materials and methods section. There seems to be a misunderstanding regarding the choice of the linear attenuation coefficient to be applied during attenuation correction in brain SPET studies. It is well known that while attenuation decreases the number of photons which can be acquired from a source, scatter will add photons. Correction of simply the number of detected photons can be performed using lower values for the narrow-beam attenuation coefficient [5] (e.g., $\mu=0.10-0.12 \mathrm{~cm}^{-1}$ rather than $\mu=0.15 \mathrm{~cm}^{-1}$ for 99mTc-labelled compounds), the most appropriate choice being dependent on the energy window setting. This empirical approach was often implemented in commercial systems in the 1990s and is considered to be an intrinsic scatter correction procedure [14]. A slightly lower value of the attenuation coefficient is used for the following reason. The full value of $\mu$ predicts how many photons will be removed from a single, narrow beam of radiation owing to the combined processes of absorption and scatter. It ignores the number of photons that can be scattered into the path from other directions. That is, it ignores the build-up caused by the broad-beam conditions of nuclear medicine imaging. Use of the actual narrowbeam value of $\mu$ without explicitly correcting for scatter will overcorrect for attenuation, and an image excessively hot in the centre will result. Although images may be cosmetically enhanced, improvements in image quantitation are only minimal with the reduced $\mu$ method of scatter correction. This is because this method assumes that scatter affects all locations in the image to the same extent. However, scatter is object and depth dependent, and a correction method which does not take this fact into account may result in large quantitation errors [15].

On the other hand, the skull thickness influences the mean effective broad-beam attenuation coefficient $\left(\mu_{\text {eff }}\right)$ by a paradoxical lowering [16]. The attenuation of the skull has been evaluated by many investigators $[3,7$, 10], all of whom have suggested the use of a lower value of $\mu_{\mathrm{eff}}$ than for a uniform soft tissue medium. The choice of the "optimal value" of the linear attenuation coefficient was studied in an elegant paper by Kemp et al. [3], where the use of effective bone and tissue attenuation coefficients to compensate $99 \mathrm{mTc}-\mathrm{HMPAO}$ brain SPET projections for attenuation resulted in images of improved uniformity and increased count density. In another study using an anthropomorphic phantom, the "best" choice of the effective linear attenuation coefficient was found to be slice dependent and reliant on the skull thickness and the methods used for attenuation and scatter corrections [4]. The deviation from the theoretical value of $0.15 \mathrm{~cm}^{-1}$ may, in all cases, be explained by non-optimal scatter corrections.

The theoretical narrow-beam attenuation coefficient is $0.148 \mathrm{~cm}^{-1}$ for the $159-\mathrm{keV}$ gamma rays of ${ }^{123} \mathrm{I}$ in water [17]. In Shiga et al.'s study, a value of $0.146 \mathrm{~cm}^{-1}$ was used, as reported in [18], for scatter-corrected images and a value of $0.10 \mathrm{~cm}^{-1}$ for images without scatter correction, a choice motivated according to the authors by a phantom study without further rationale and justification [1]. Van Laere et al. [9] used an attenuation coefficient of $1.05 \mathrm{~cm}^{-1}$ determined from experimental studies using the 3D Hoffman brain phantom and $0.09 \mathrm{~cm}^{-1}$ for clinical studies [10], indicating that results obtained from phantom studies cannot be extrapolated directly for application to human data.

With respect to the title of the paper, the reader would expect an investigation of the effect of scatter correction on regional activity distribution of brain perfusion SPET using SPM analysis, whereas from the description provided, it looks as if two scatter correction schemes are 
compared, i.e. the empirical reduced $\mu$ method and the energy-based scatter correction method. If the motivation is to assess the effect of scatter correction, the reasons for modifying the linear attenuation coefficient between the two data sets are not clear.

The other aspect that deserves special attention is the choice of the attenuation correction method. It is generally well accepted that transmission-based non-uniform attenuation correction can supply more accurate absolute quantification; however, whether it provides specific benefits in the routine clinical practice of functional brain imaging is still the subject of debate [10, 19]. Some authors have reported that uniform attenuationcorrected studies provide unreliable regional estimates of tracer activity [6]. Others, using Monte Carlo simulation studies of the Zubal brain phantom, have shown that an accurate attenuation map is crucial for absolute quantitation, but less of an issue in relative quantitation in brain SPET [20]. In the Discussion section, Shiga et al. considered the various methods that may be used for attenuation correction and argue that the attenuation map will have only a minimal effect on the conclusions drawn from their study. They recognise that transmission-based attenuation correction is the clinical "gold standard" without offering further comments on the reasons why it was not used in their study. Moreover, estimation of the attenuation map from a segmented reconstruction of a lower-energy Compton scatter window image was reported as the next most accurate clinical method and can be reliably used when transmission scanning cannot be used [6].

The other concern is related to the energy window settings for application of the triple-energy scatter correction technique. As the authors point out, this method increases the noise and it is well known that SPM analysis is very sensitive to statistical noise. A Monte Carlo investigation of the method by Ljungberg et al. [21] questioned the utility of the upper (right) scatter window for ${ }^{99} \mathrm{Tc}$, noting that using the right scatter window might make the TEW method more susceptible to noise. In a related study, it has been shown that the scatter component is overestimated by $18 \%$ when both windows are used and underestimated by $14 \%$ when only the lower window is used [22]. Neglecting the higher scatter window has also been recommended to avoid increasing statistical noise [23]. The energy settings used in the study by Shiga et al. were $160 \mathrm{keV}$ peak with $24 \%$ width for the main window and $3 \%$ width for the scatter rejection window. This means that the upper scatter rejection window was neglected, an assumption with proven validity for ${ }^{99} \mathrm{mTc}$ but not for ${ }^{123} \mathrm{I}$ [18]. The optimal choice of the acquisition window for ${ }^{123}$ I-labelled compounds taking into account its decay scheme and the impact of downscatter from high-energy photons (e.g. $520 \mathrm{keV}$ ) was not addressed and deserves further discussion as well.

In summary, we want to stress the importance of attenuation and scatter corrections on regional activity dis- tribution in quantitative brain SPET and the need for rigour in describing the methods used so as to ensure both the clarity and the reproducibility of published work.

\section{Habib Zaidi (르), Marie-Louise Montandon}

Division of Nuclear Medicine,

Geneva University Hospital,

1211 Geneva 4, Switzerland

e-mail: habib.zaidi@hcuge.ch

Tel.: +41-22-3737358

Fax: $+41-22-3727169$

\section{References}

1. Shiga T, Kubo N, Takano A, et al. The effect of scatter correction on ${ }^{123}$ I-IMP brain perfusion SPET with the triple energy window method in normal subjects using SPM analysis. Eur J Nucl Med 2002; 29:342-345.

2. Zaidi H. Comparative evaluation of scatter correction techniques in 3D positron emission tomography. Eur J Nucl Med 2000; 27:1813-1826.

3. Kemp BJ, Prato FS, Dean GW, Nicholson RL, Reese L. Correction for attenuation in technetium-99m-HMPAO SPECT brain imaging. J Nucl Med 1992; 33:1875-1880.

4. Arlig A, Gustafsson A, Jacobsson L, Ljungberg M, Wikkelso C. Attenuation correction in quantitative SPECT of cerebral blood flow: a Monte Carlo study. Phys Med Biol 2000; 45:3847-3859.

5. Harris CC, Greer KL, Jaszczak RJ, et al. Tc-99m attenuation coefficients in water-filled phantoms determined with gamma cameras. Med Phys 1984; 11:681-685.

6. Licho R, Glick SJ, Xia W, et al. Attenuation compensation in 99mTc SPECT brain imaging: a comparison of the use of attenuation maps derived from transmission versus emission data in normal scans. J Nucl Med 1999; 40:456-463.

7. Stodilka RZ, Kemp BJ, Prato FS, Nicholson RL. Importance of bone attenuation in brain SPECT quantification. $J \mathrm{Nucl}$ Med 1998; 39:190-197.

8. Stodilka RZ, Kemp BJ, Prato FS, et al. Scatter and attenuation correction for brain SPECT using attenuation distributions inferred from a head atlas. J Nucl Med 2000; 41:1569-1578.

9. Van_Laere K, Koole M, Kauppinen T, et al. Nonuniform transmission in brain SPECT using ${ }^{201} \mathrm{Tl},{ }^{153} \mathrm{Gd}$, and ${ }^{99 \mathrm{~m} T c}$ static line sources: anthropomorphic dosimetry studies and influence on brain quantification. J Nucl Med 2000; 41:2051-2062.

10. Van_Laere K, Koole M, Versijpt J, Dierckx R. Non-uniform versus uniform attenuation correction in brain perfusion SPET of healthy volunteers. Eur J Nucl Med 2001; 28:90-98.

11. Ichihara T, Motomura N, Ogawa K, et al. Evaluation of SPET quantification of simultaneous emission and transmission imaging of the brain using a multidetector SPET system with the TEW scatter compensation method and fan-beam collimation. Eur J Nucl Med 1996; 23:1292-1299.

12. Ogasawara K, Hashimoto J, Ogawa K, et al. Simultaneous acquisition of iodine-123 emission and technetium-99m transmission data for quantitative brain single-photon emission tomographic imaging. Eur J Nucl Med 1998; 25:15371544.

13. Rajeevan N, Zubal IG, Ramsby SQ, et al. Significance of nonuniform attenuation correction in quantitative brain SPECT imaging. J Nucl Med 1998; 39:1719-1726. 
14. Zaidi H. Quantitative SPECT: recent developments in detector response, attenuation and scatter correction techniques. Phys Med 1996; 12:101-117.

15. Zaidi H. Scatter modelling and correction strategies in fully 3D PET. Nucl Med Commun 2001; 22:1181-1184.

16. Nicholson R, Doherty M, Wilkins K, Prato F. Paradoxical effects of the skull on attenuation correction requirements for brain SPECT. J Nucl Med 1988; 29:1316.

17. Berger MJ, Hubbell JH. "XCOM: Photon cross sections on a personal computer." NBSIR 87-3597 (National Bureau of Standards (US), 1987).

18. Ichihara T, Ogawa K, Motomura N, Kubo A, Hashimoto S. Compton scatter compensation using the triple-energy window method for single- and dual-isotope SPECT. J Nucl Med 1993; 34:2216-2221.

19. Zaidi H, Laemmli C, Allaoua M, Gries PD, Slosman O. Optimizing attenuation correction in clinical cerebral 3D PET: which method to use? J Nucl Med 2001; 42:P195-P196.

20. El Fakhri G, Moore SC, Kijewski MF. Impact of the attenuation map on relative and absolute quantitation in brain SPECT. J Nucl Med 2000; 41:61P.

21. Ljungberg M, King MA, Hademenos GJ, Strand SE. Comparison of four scatter correction methods using Monte Carlo simulated source distributions. J Nucl Med 1994; 35:143-151.

22. Buvat I, Rodriguez-Villafuerte M, Todd-Pokropek A, Benali H, Di Paola R. Comparative assessment of nine scatter correction methods based on spectral analysis using Monte Carlo simulations. J Nucl Med 1995; 36:1476-1488.

23. Ogawa K, Harata Y, Ichihara T, Kubo A, Hashimoto S. A practical method for position-dependent Compton-scattered correction in single photon emission CT. IEEE Trans Med Imaging 1991; 10:408-412.

Eur J Nucl Med (2002) 29:969-970

DOI 10.1007/s00259-002-0841-2

Published online: 25 May 2002

(C) Springer-Verlag 2002

\section{Reply}

Dear Sir,

We read with interest the letter from Drs. Zaidi and Montandon. We appreciate their valuable comments, but there seems to have been a lot of misunderstanding regarding our paper.

First, the aim of our study was to investigate the difference in ${ }^{123}$ I-IMP distribution using brain perfusion SPET with and without scatter correction, not to investigate the quantitative difference between them [1]. As mentioned in the Introduction to our paper, there is an excellent paper by Iida et al. [2] on the quantitative difference in regional cerebral blood flow with different scatter and attenuation correction methods based on re- gion of interest analysis. In our paper, we compared images obtained using (a) Chang's attenuation correction method [uniform $\mu$ map using a broad $\mu$ value $\left.\left(0.10 \mathrm{~cm}^{-1}\right)\right]$ without scatter correction, and (b) Chang's attenuation correction method [uniform $\mu$ map using a narrow $\mu$ value $\left.\left(0.146 \mathrm{~cm}^{-1}\right)\right]$ with triple energy window scatter correction.

Another misunderstanding of Drs. Zaidi and Montandon is that the upper scatter rejection window was neglected. We applied the triple energy window (TEW) scatter correction method in our study, not the dual window method. We used three energy windows: one main window and upper and lower scatter rejection windows. In fact, the upper rejection window may be neglected on condition that a ${ }^{99 \mathrm{~m} T c-l a b e l l e d ~ a g e n t ~ i s ~ u s e d ~[3] . ~ B u v a t ~ e t ~}$ al. reported that the TEW method leads to overestimation when using 99m Tc [4]. In our study, we used ${ }^{123} \mathrm{I}-$ IMP and the aforementioned findings were therefore inapplicable; accordingly, we did not refer to those papers.

As already indicated, for attenuation correction we applied Chang's method. It is the best way of correcting images with a $\mu$ map obtained from the transmission scan. However, as described by Licho et al. [5], the transmission imaging technique produces an estimate of the narrow-beam attenuation coefficient map. Therefore, scatter correction of emission data is necessary. Attenuation correction with the measured $\mu$ map definitely leads to overcorrection, especially in the central part of objects, without scatter correction. On the other hand, Iida et al. [2] reported that reconstruction using a uniform $\mu$ attenuation map and a narrow $\mu$ with scatter correction could be used to assess regional cerebral blood flow with little loss of accuracy in most brain regions. In our study, since we compared images with and without scatter correction, conditions had to be matched as closely as possible. Therefore, we chose Chang's attenuation correction method. However, without transmission, errors may occur when determining the contour of the constant $\mu$ attenuation map. In this study, since the contour of the constant $\mu$ attenuation map was determined using emission data both with and without scatter correction, the contour was almost same between the two images. As we described, two images were compared within each subject. The errors that were derived from the uniform $\mu$ map made from almost the same contour of those two images were considered to be almost the same. Therefore, the errors should have been cancelled out in the statistical procedure.

Licho et al. [5] reported the estimation of an attenuation map from segmented reconstruction of a low-energy Compton scatter window image when using $99 \mathrm{mTc}-$ HMPAO SPET. We used 123I-IMP for brain perfusion SPET, and to our knowledge there has been no report on the optimal window range and energy peak for estimation of the attenuation map with ${ }^{123}$ I-IMP brain perfusion SPET. We therefore thought that the application of the aforementioned method to our study was not appropriate. 